\title{
Diurnal and Nocturnal Activity Patterns of Invasive Wild Boar (Sus scrofa) in Saskatchewan, Canada
}

\author{
Kathryn Stolle ${ }^{1}$, Floris M. van Beest ${ }^{1,2}$, Eric VANDER WaL ${ }^{1,3,4}$, AND Ryan K. Brook ${ }^{1,5,6}$ \\ ${ }^{1}$ Department of Animal and Poultry Science, College of Agriculture and Bioresources, University of Saskatchewan, 51 Campus \\ Drive, Saskatoon, Saskatchewan S7N 5E2 Canada \\ ${ }^{2}$ Department of Bioscience, Aarhus University, Frederiksborgvej 399, 4000 Roskilde, Denmark \\ ${ }^{3}$ Département de biologie, Université de Sherbrooke, 2500, boulevard de l'université, Sherbrooke, Québec J1K 2R1 Canada \\ ${ }^{4}$ Department of Biology, Memorial University, St. John's, Newfoundland A1B 3X9 Canada \\ ${ }^{5}$ Indigenous Land Management Institute, College of Agriculture and Bioresources, University of Saskatchewan, 51 Campus \\ Drive, Saskatoon, Saskatchewan S7N 5E2 Canada \\ ${ }^{6}$ Corresponding author: ryan.brook@usask.ca
}

Stolle, Kathryn, Floris M. van Beest, Eric Vander Wal, and Ryan K. Brook. 2015. Diurnal and nocturnal activity patterns of invasive Wild Boar (Sus scrofa) in Saskatchewan, Canada. Canadian Field-Naturalist 129(1): 76-79.

The Wild Boar (Sus scrofa) is invasive in western Canada and poses a significant ecological and socio-economic threat over much of the country. We sought to quantify their presence and to determine when they are most active and whether their activity patterns are influenced by group size. Digital trail cameras $(n=18)$ were placed in a stratified design in the four most dominant habitat types of central Saskatchewan, Canada, and activated between December 2011 and June 2013 for a total of 5715 trap-days. In 71175 photographs, we obtained 22 individual visits of Wild Boars to the trail cameras. We found no differences in activity between night (1900-0700; 59\% of all detections) and day (0701-1859; 41\% of detections), and we did not detect any effect of group size. Ongoing monitoring will be required to determine changing activity patterns in response to changing hunting pressure as Wild Boar continue to expand across Canada.

Key Words: diurnal activity; nocturnal activity; Wild Boar; Sus scrofa; feral swine; invasive species; Saskatchewan; trail camera

\section{Introduction}

The Wild Boar (Sus scrofa), which is native to Eurasia, has been introduced into North America on several occasions (Witmer et al. 2003; Barrios-Garcia and Ballari 2012). It is a successful invader because it easily adapts to novel food sources, is highly fecund, and is vagile (Witmer et al. 2003; Barrios-Garcia and Ballari 2012). Wild Boar are known to cause considerable environmental and agricultural damage (Campbell and Long 2010; Barrios-Garcia and Ballari 2012) and spread disease (Witmer et al. 2003; Leiser et al. 2013).

Little is known about the Wild Boar in Canada, including spatial distribution, abundance, and activity patterns. Their naturalization followed their use in specialized livestock farms, which has a history of less than 35 years (Brook and van Beest 2014). What is apparent, however, is that they now exist in viable populations across the Canadian Prairies (Brook and van Beest 2014). Despite the imminent ecological and socioeconomic threat they pose, management (or eradication) efforts remain at best ineffective, at worst some management efforts such as use of bounties likely help disperse animals widely and increase population growth. The challenge is further exacerbated by the confusing taxonomic of the species, where both domestic pigs $(S$. scrofa domestica) and Eurasian Wild Boar (primarily S. s. scrofa but may also include other subspecies) are both the same species and hybrids between these and other subspecies occur in the United States and likely also occur in Canada (Wilson and DeeAnn 2005). These hybrids may have even higher reproductive success but there is little information in Canada to clarify their taxonomy or the implications of the hybridization.

Our objective was to quantify the presence and activity patterns of this newly invasive species in Canada. In particular, we aimed to determine when Wild Boar are most active and whether their activity patterns are influenced by group size. We did this using a system of remote trail cameras. As Wild Boar are a relatively new species on the Canadian Prairies, we suspected that they exist at low densities, even though they are widespread in distribution (Brook and van Beest 2014).

\section{Study Area}

Our study area $\left(275 \mathrm{~km}^{2} ; 52^{\circ} 37^{\prime} \mathrm{N}, 104^{\circ} 51^{\prime} \mathrm{W}\right)$ is in central Saskatchewan, Canada, near the town of St. Breiux. It is part of the transition area from the Prairie Ecozone in the south to the Boreal Plains Ecozone in the north and is referred to as the Prairie Parklands. The area is a complex mix of annual cropland and perennial forage farmland intermixed with fragmented native deciduous aspen forest, grassland, and wetland. The study area is a part of the prairie pothole region of the Great Plains, which is characterized by thousands of shallow pothole wetlands. Human density is low in the rural landscape: 0.74 people $/ \mathrm{km}^{2}$ and 0.27 households/ $\mathrm{km}^{2}$. The only town in the study area, St. Brieux, has a population of 590 people (Statistics Canada 2011). Hunting of Wild Boar in the study area is unregulated by the province of Saskatchewan, and no population estimates or metrics of annual harvest are recorded. 


\section{Methods}

Between December 2011 and June 2013, we deployed 18 HyperFire 900 camera units (Reconyx Inc., Holmen, Wisconsin, USA) in four dominant habitat types: deciduous forest $(n=5)$, grasslands $(n=4)$, pasture-croplands $(n=5)$, and wetlands $(n=4)$. Cameras were placed within $50 \mathrm{~m}$ of a preselected location determined by a stratified random design and along game trails or open space if present. Each unit was attached to a tree or fence post, $100 \mathrm{~cm}$ above the ground facing in the direction with the least amount of vegetation cover (no vegetation was cleared except for twigs and leaves less than $1 \mathrm{~m}$ from the camera lens). We did not place bait in the vicinity of the cameras as we wished to detect natural behaviour. We programmed all cameras at the normal sensitivity setting (three photos at 1-s intervals triggered by an animal passing across the passive infrared motion detector in each camera), as this was found to be reliable during test trials (unpublished data). The metadata associated with each photo included a unique number, the time of day, and date.

All photographs were screened to identify those that contained Wild Boar. Most photos were of native wild- life common in the study area. For each non-native Wild Boar photo, we recorded the observed group size. In many cases, multiple photos were taken of an animal at a camera over several seconds or minutes, so regardless of the number of photos taken, we defined one individual "visit" to a camera as being from the first appearance of the animal to its disappearance from the camera over a period of 15 minutes. The largest group size recorded during each visit was assumed to be the final group size.

We compared frequency of visits between expected (i.e., randomly distributed) and observed numbers of Wild Boar for each hour of the day using a $\chi^{2}$ test, and compared observed group sizes using a Mann-Whitney $U$-test.

\section{Results}

Between December 2011 and June 2013, we obtained 71175 photos (5715 trap-days, calculated as the total number of camera traps * the total number of days the cameras were deployed) (see Figure 1 for samples). Between August 2012 and June 2013, three trail cameras and/or their photo cards were stolen and some data
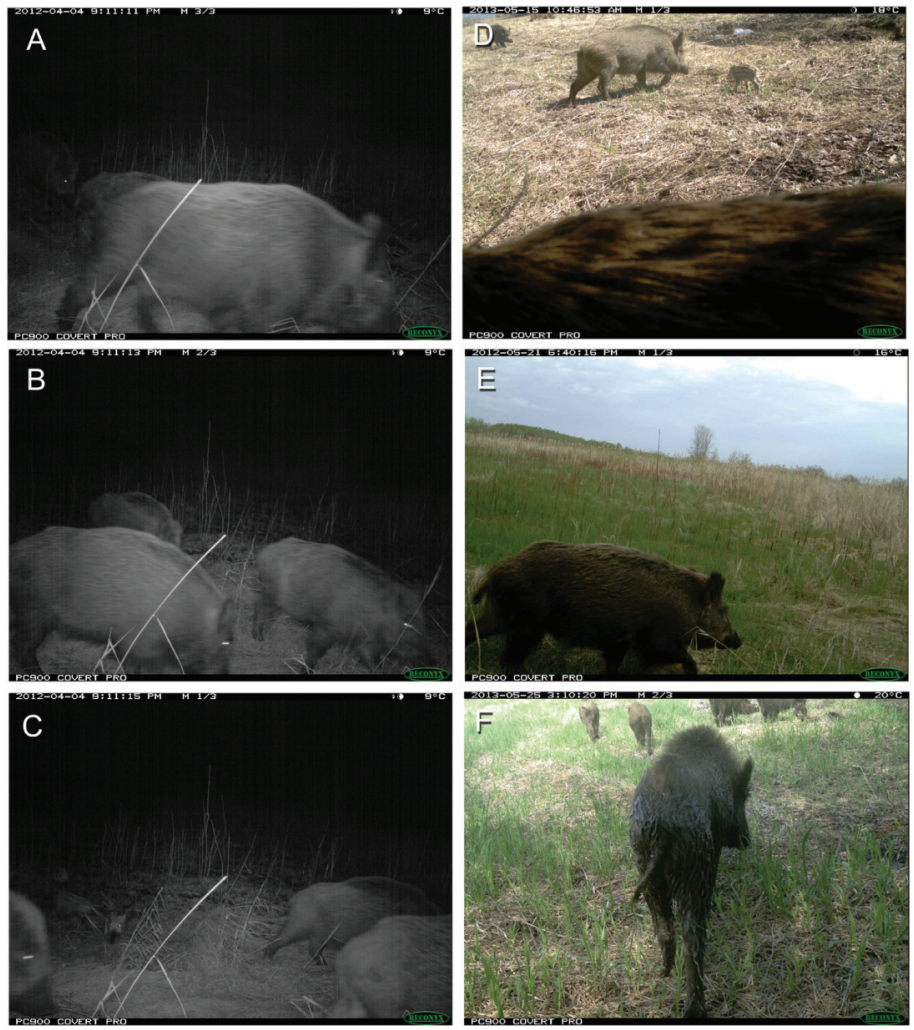

Figure 1. Photographs (A-F) taken by four trail cameras near St. Brieux, Saskatchewan, Canada, in 2012 and 2013 showing an adult female Wild Boar walking past the camera (A), with a litter of her young that are approximately 4 months old characterized by their size and lack of piglet striping of pale lines (B), followed by another litter of smaller 'squeaker' piglets approximately 3 weeks of age characterized as very small in size and having cream coloured stripes (C), all of these occurring at night, an image of a different group of Wild Boar during the daytime that includes young piglets (D), an image of a solitary animal in daylight (E), and a sounder of larger Wild Boar during the day (F). 
were lost. We captured 22 individual visits of Wild Boar to trail cameras during the study period. In these visits we counted a minimum of 60 individual animals. In one instance, two Wild Boars were detected by a camera for approximately $2 \mathrm{~h}$ continuously. Another group joined the original group after the $2 \mathrm{~h}$ had elapsed. We considered the arrival of the second group to be a separate visit. More than half of the visits $(59.1 \%)$ were of a single individual. Group size within a frame ranged from one to nine. The first Wild Boar was sighted on 15 December 2011 and the last on 20 May 2013. The number of visits varied considerably among months (Table 1).

TABLE 1. Sightings of Wild Boar (Sus scrofa) using trail cameras from December 2011 to June 2013 near St. Brieux, Saskatchewan, Canada.

\begin{tabular}{lcccc}
\hline \hline Month & $\begin{array}{c}\text { No. Boar } \\
\text { visits }\end{array}$ & $\begin{array}{c}\text { No. in } \\
\text { Wetland }\end{array}$ & $\begin{array}{c}\text { No. in } \\
\text { Cropland }\end{array}$ & $\begin{array}{c}\text { No. in } \\
\text { Deciduous } \\
\text { Forest }\end{array}$ \\
\hline January & 0 & 0 & 0 & 0 \\
February & 0 & 0 & 0 & 0 \\
March & 3 & 2 & 1 & 0 \\
April & 7 & 3 & 4 & 0 \\
May & 2 & 2 & 0 & 0 \\
June & 0 & 0 & 0 & 0 \\
July & 1 & 0 & 0 & 1 \\
August & 0 & 0 & 0 & 0 \\
September & 5 & 5 & 0 & 0 \\
October & 1 & 1 & 0 & 0 \\
November & 0 & 0 & 0 & 0 \\
December & 3 & 1 & 2 & 0 \\
\hline \hline
\end{tabular}

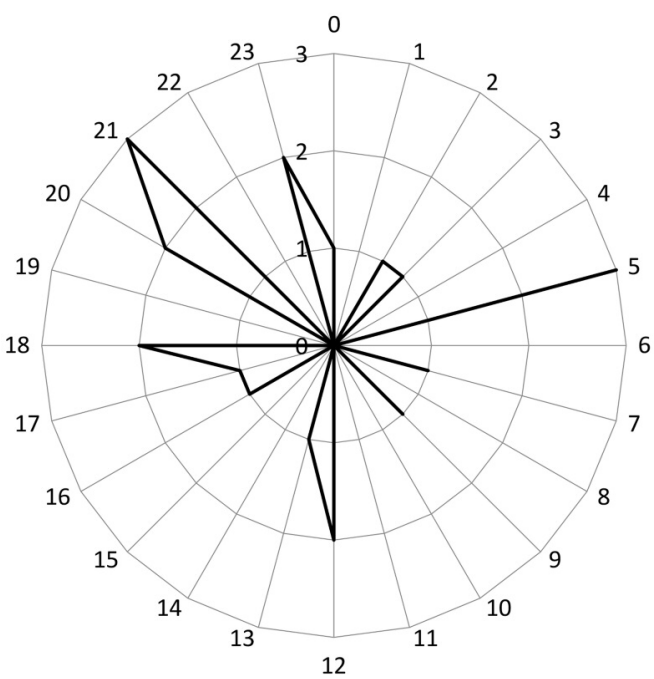

Figure 2. Time of visits of Wild Boar (Sus scrofa) at trail cameras from December 2011 to June 2013 near St. Brieux, Saskatchewan, Canada. The number of visits, from 0 to 3 , is indicated by the distance from the centre of the circle; the hour of day is shown on the circumference.
There was no difference between expected (i.e., randomly distributed) and observed numbers of Wild Boar visits at each hour of the day $\left(\chi^{2}=7.0, P=0.072\right)$. Nocturnal (1900-0700) and diurnal (0701-1859) group sizes did not vary (Mann-Whitney $U=51.5, P=0.518$ ). The largest number of sightings occurred at $2400 \mathrm{~h}$ and half of the sightings $(50 \%)$ occurred between 2000 and $2400 ; 59.1 \%$ of detections occurred at night (19000700; Figure 2). Wild Boar observations occurred in three habitats: wetland (64\%), pasture-cropland (32\%), and deciduous forest (4\%).

\section{Discussion}

Observations of Wild Boar visits detected by trail cameras in central Saskatchewan, Canada, did not differ between day and night and group size, nor did visits vary significantly according to time of day. Although our cameras were active for 1.5 years, the number of Wild Boar visits to trail cameras was low. Because the efficacy of our cameras was high, it was unlikely that we failed to detect Wild Boar. Therefore, it seems that Wild Boar population densities are relatively low, at least in our study area. Nevertheless, after randomly distributing camera traps over a landscape, we managed to detect their presence and obtain photographs of successful reproductions in the wild (Figure 1) and one short series of continuous photos that capture a group (often called a 'sounder') led by an adult female (panel A, Figure 1) with one litter of juvenile piglets approximately 4 months old characterized by their size and lack of piglet striping of pale lines (panel B, Figure 1) and a second litter of 'squeaker' piglets approximately 3 weeks of age characterized as very small in size and having cream coloured stripes (panel C, Figure 1).

We do not know when Wild Boar first colonized our study area. However, it seems a sufficiently long period (i.e., generations) for them to have acclimated to local human presence by foraging and moving during both day and night. Wild Boar typically flee when approached by humans and are known to alter their activity patterns based on human activity (Singer et al. 1981). The distance to human dwellings also affects the activity patterns of Wild Boar (Keuling et al. 2008), although the density of human dwellings is very low in our study area.

Within their native range (e.g., Italy), Wild Boar are most active between 1700 and 0700 (Russo et al. 1997). Where boar are invasive, activity patterns vary; for example, in the United States, such variation exists among states (Campbell and Long 2010). Although hunting by humans is considered a key driver of increased nocturnal activity, some studies have found that hunting increases daytime activity while others report that nocturnal activity increases (Keuling et al. 2008). These observations of activity patterns are relevant to possible population control efforts, as visual detection of Wild Boar is more challenging at night and firearms 
regulations in western Canada do not allow discharge of a firearm at night. Thus, ongoing monitoring will be critical to understanding changes in activity patterns in response to management actions.

\section{Acknowledgements}

This work was funded by the University of Saskatchewan through the Natural Sciences and Engineering Research Council of Canada's President's Fund, the Cyril Capling Trust Fund, and a research start-up grant to R. K. B. We thank Caitlin Labach for logistic and field support. The Wildlife Ecology and Community Engagement Lab provided ongoing input and support. We especially acknowledge the many rural landowners in Saskatchewan who kindly gave us permission to set up cameras on their land.

\section{Literature Cited}

Barrios-Garcia, M. N., and S. A. Ballari. 2012. Impact of swine (Sus scrofa) in its introduced and native range: a review. Biological Invasions 14: 2283-2300.

Brook, R. K., and F. M. van Beest. 2014. Feral wild boar distribution and perceptions of risk on the central Canadian Prairies. Wildlife Society Bulletin 38: 486-494.

Campbell, T. A., and D. B. Long. 2010. Activity patterns of swines (Sus scrofa) in southern Texas. Southwest Naturalist 55: 564-600.
Keuling, O., N. Stier, and M. Roth. 2008. How does hunting influence activity and spatial usage in swine Sus scrofa L.?. European Journal of Wildlife Research 54: 729-737.

Leiser, O. P., J. L. Corn, B. S. Schmit, P. S. Keim, and J. T. Foster. 2013. Feral swine brucellosis in the United States and prospective genomic techniques for disease epidemiology. Veterinary Microbiology 166: 1-10.

Russo, L., G. Massei, and P. V. Genov. 1997. Daily home range and activity of swine in a Mediterranean area free from hunting. Ethology, Ecology and Evolution 9: 287294.

Singer, F. J., D. K. Otto, A. R. Tipton, and C. P. Hable. 1981. Home ranges, movements, and habitat use of European swine in Tennessee. Journal of Wildlife Management 45: 343-353.

Statistics Canada. 2011. 2011 community profiles, Canada 2011 Census. Accessed 10 November 2014. www.statcan .gc.ca.

Wilson, D. E., and D. M. Reeder. 2005. Mammal Species of the World - A Taxonomic and Geographic Reference. Third edition. ISBN 0801882214.

Witmer, G. W., R. B. Sanders, and A. C Taft. 2003. Feral swine - are they a disease threat to livestock in the United States? Staff publications paper 292. United States Department of Agriculture, National Wildlife Research Center, Lincoln, Nebraska, USA.

Received 30 April 2014

Accepted 4 November 2014 